\title{
Pemphigus autoantibodies generated through somatic mutations target the desmoglein-3 cis-interface
}

\author{
Giovanni Di Zenzo,, ${ }^{1}$ Giulia Di Lullo, ${ }^{2}$ Davide Corti, ${ }^{2,3}$ Valentina Calabresi, ${ }^{1}$ Anna Sinistro, ${ }^{1}$ \\ Fabrizia Vanzetta, ${ }^{3}$ Biagio Didona, ${ }^{4}$ Giuseppe Cianchini, ${ }^{5}$ Michael Hertl, ${ }^{6}$ Rudiger Eming, ${ }^{6}$ \\ Masayuki Amagai,7 Bungo Ohyama, ${ }^{8}$ Takashi Hashimoto, ${ }^{8}$ Jerry Sloostra, ${ }^{9}$ \\ Federica Sallusto,2 Giovanna Zambruno, ${ }^{1}$ and Antonio Lanzavecchia 2,10
}

\begin{abstract}
${ }^{1}$ Molecular and Cell Biology Laboratory, Istituto Dermopatico dell'Immacolata, Istituto di Ricovero e Cura a Carattere Scientifico (IDI-IRCCS), Rome, Italy. 2Institute for Research in Biomedicine (IRB), Bellinzona, Switzerland. 3Humabs BioMed SA, Bellinzona, Switzerland. 4 I Dermatology Division, and ${ }^{5} \mathrm{~V}$ Dermatology Division, IDI-IRCCS, Rome, Italy. ${ }^{6}$ Department of Dermatology and Allergology, Philipps University, Marburg, Germany. ${ }^{7}$ Department of Dermatology, Keio University School of Medicine, Tokyo, Japan. ${ }^{8}$ Department of Dermatology, Kurume University School of Medicine and Kurume University Institute of Cutaneous Cell Biology, Kurume, Japan. ${ }^{9}$ Pepscan Presto, Lelystad, The Netherlands.

${ }^{10}$ Institute of Microbiology, Eidgenössische Technische Hochschule (ETH), Zürich, Switzerland.
\end{abstract}

\begin{abstract}
Pemphigus vulgaris (PV) is an autoimmune blistering disease of skin and mucous membranes caused by autoantibodies to the desmoglein (DSG) family proteins DSG3 and DSG1, leading to loss of keratinocyte cell adhesion. To learn more about pathogenic PV autoantibodies, we isolated 15 IgG antibodies specific for DSG3 from $2 \mathrm{PV}$ patients. Three antibodies disrupted keratinocyte monolayers in vitro, and 2 were pathogenic in a passive transfer model in neonatal mice. The epitopes recognized by the pathogenic antibodies were mapped to the DSG3 extracellular 1 (EC1) and EC2 subdomains, regions involved in cis-adhesive interactions. Using a site-specific serological assay, we found that the cis-adhesive interface on EC1 recognized by the pathogenic antibody PVA224 is the primary target of the autoantibodies present in the serum of PV patients. The autoantibodies isolated used different heavy- and light-chain variable region genes and carried high levels of somatic mutations in complementary-determining regions, consistent with antigenic selection. Remarkably, binding to DSG3 was lost when somatic mutations were reverted to the germline sequence. These findings identify the cis-adhesive interface of DSG3 as the immunodominant region targeted by pathogenic antibodies in PV and indicate that autoreactivity relies on somatic mutations generated in the response to an antigen unrelated to DSG3.
\end{abstract}

\section{Introduction}

Pemphigus vulgaris (PV) is a life-threatening autoimmune blistering disease of skin and mucous membranes caused by autoantibodies that bind to the cadherin-type cell-cell adhesion molecules desmoglein 3 (DSG3) and DSG1, the main constituents of desmosomes, and cause the loss of keratinocyte cell adhesion (1). The critical role of autoantibodies in PV pathogenesis is supported by the observations that the disease activity correlates with anti-DSG3 antibody titers (2), that newborns of mothers with active PV exhibit blisters caused by the placental transfer of maternal antibodies (3), and that pemphigus-like lesions are induced in neonatal mice by passive transfer of anti-DSG3 IgG from PV patients (4).

In the skin, DSG3 is mainly expressed in the basal and suprabasal layers (5), while DSG1 is predominantly expressed in the upper epidermal layers. In contrast, in noncornified stratified epithelia, such as the oral mucosa, DSG3 is highly expressed throughout the epithelium, while DSG1 is expressed at a much lower level. The differential expression pattern of DSG1 and DSG3 is respon-

Authorship note: Giovanni Di Zenzo, Giulia Di Lullo, and Davide Corti contributed equally to this work.

Conflict of interest: Antonio Lanzavecchia is the scientific founder of Humabs BioMed. Davide Corti and Fabrizia Vanzetta are currently employees of Humabs BioMed. Antonio Lanzavecchia and Federica Sallusto hold shares in Humabs BioMed. Citation for this article: JClin Invest. 2012;122(10):3781-3790. doi:10.1172/JCI64413. sible for clinical variants of pemphigus (6): antibodies to DSG3 are present in the mucosal form, while antibodies to both DSG3 and DSG1 are associated with mucocutaneous lesions (7).

DSG3 is a calcium-binding membrane glycoprotein with an extracellular domain comprising 5 distinct subdomains (EC1EC5), and it is synthesized as proprotein, which is processed in the Golgi apparatus by removal of a propeptide before transporting to the cell surface. The cleavage of the propeptide occurs upstream of a conserved tryptophan residue in the EC1 subdomain, unmasking residues critical for the formation of homophilic interactions with DSG3 on opposing cells $(8,9)$. Several studies have shown that polyclonal antibodies in PV serum react primarily with the aminoterminus of DSG3 in the EC1 and EC2 subdomains (amino acids 1-161) (10,11).

The isolation of pathogenic mAbs is instrumental for addressing questions as to the mechanism that induces the autoreactive response and drives blister formation in PV patients. Amagai and coworkers isolated from an active mouse model of PV a pathogenic antibody, AK23, which causes loss of cell adhesion by binding to the EC1 subdomain of DSG3 that is involved in the formation of the trans-adhesive interface $(12,13)$. A number of human anti-DSG pathogenic and nonpathogenic mAbs were isolated as single-chain variable-region fragments (scFvs) from a PV patient (14). Similarly to the AK23 mAb, the pathogenic activity of these human antibodies was mapped to the aminoterminal region of EC1, which is masked by the propeptide $(9,13,14)$. Taken together, 


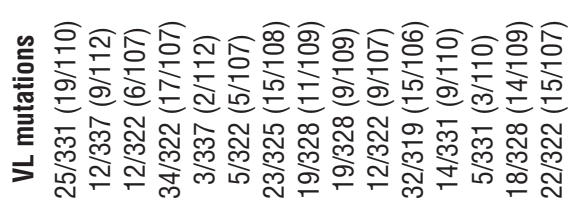

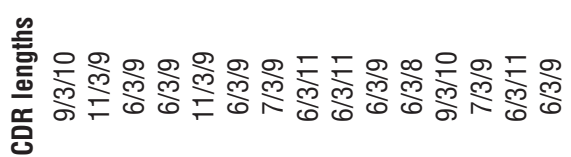

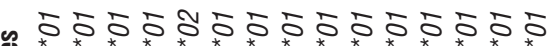

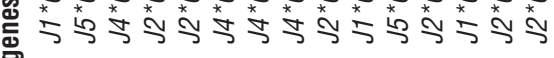
3

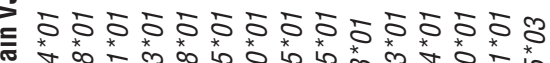

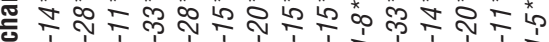
งัง
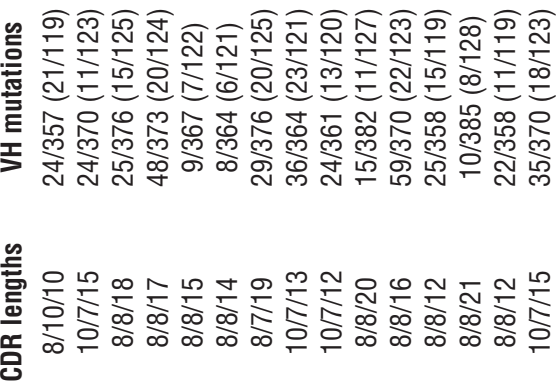

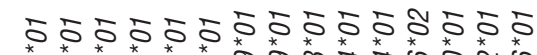

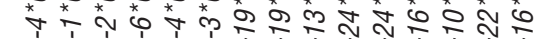

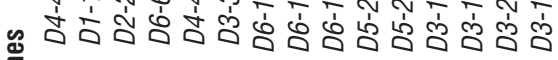
票

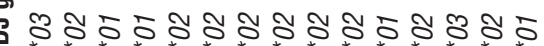

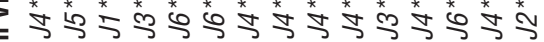
돈

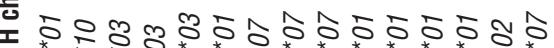

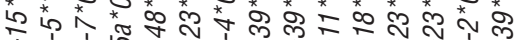

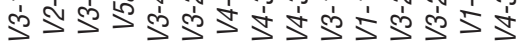

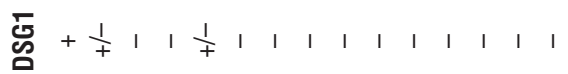

ஜ

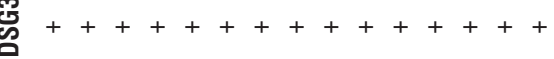

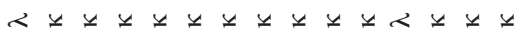

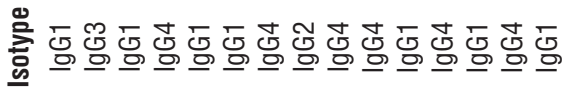

the human and mouse data suggest that pathogenic antibodies bind primarily to EC1 and disrupt the keratinocyte adhesion by interfering with the trans-adhesive interface of DSG3.

In this study, we isolated from $2 \mathrm{PV}$ patients several IgG autoantibodies that bind DSG3. These antibodies carried high levels of somatic mutations that were required for binding to DSG3. The epitopes recognized by 3 pathogenic antibodies were mapped to the EC1 and EC2 subdomains in regions that are expected to be involved in cis-adhesive interactions. This region was found to be the primary target of serum autoantibodies in PV patients. These results identify the cis-adhesive interface as the immunodominant region targeted by pathogenic antibodies in PV and suggest that autoreactivity relies on somatic mutations triggered by an unrelated antigen.

\section{Results}

Isolation and characterization of DSG3-specific antibodies from PV patients. Peripheral blood samples were collected from 2 patients with mucocutaneous PV: one with long-lasting steroid-resistant disease (PVA) and the other prior to treatment initiation (PVB). $\mathrm{IgG}^{+}$memory $\mathrm{B}$ cells were isolated by a combination of magneticand fluorescence-activated cell sorting, seeded in 96-well microplates, and immortalized with EBV in the presence of irradiated mononuclear cells and oligodeoxynucleotides containing $\mathrm{CPG}$ motifs, as previously described (15). Culture supernatants were screened using DSG3-ELISA and indirect immunofluorescence (IF) staining of live keratinocyte monolayers. Cultures that produced specific antibodies were cloned by limiting dilution, and 15 independent clones were isolated and characterized (Table 1). Three of the 6 antibodies from patient PVA and all the 9 antibodies isolated from patient PVB bound to DSG3, while 3 antibodies from PVA crossreacted with both DSG3 and DSG1. All antibodies showed the characteristic fishnet intercellular staining of epithelial cells on human skin and monkey esophagus (Figure 1A and data not shown). In addition, 11 out of the 15 antibodies failed to bind to DSG3 in the presence of EDTA, as assessed by IF and ELISA (Figure 1, B and C), consistent with the recognition of calciumdependent conformational epitopes.

Consistent with previous reports $(16,17)$, the antibodies were primarily $\operatorname{IgG} 1$ or IgG4, although IgG2 and IgG3 antibodies were also isolated. The antibodies used different genes encoding the immunoglobulin variable regions of heavy (VH) and light (VL) chains, with a slight bias toward $V H 3$ and $V H 4$, carried heavy chain complementarity-determining region 3 (H-CDR3) and light chain complementarity-determining region 3 (L-CDR3) of different lengths (10-21 and 8-11 amino acids, respectively), and had a variable load of somatic mutations (Table 1). A higher level of replacing mutations was found in CDRs of both $\mathrm{VH}$ and $\mathrm{VL}$ (Supplemental Figure 1, A and B; supplemental material available online with this article; doi:10.1172/JCI64413DS1). The somatic mutations in $\mathrm{VH}$ and $\mathrm{VL}$ gene segments were analyzed using the implemented Focused binomial test and the Global binomial test $(18,19)$, which compare the observed frequency of replacement mutations with their expected frequency under the null hypothesis of no selection. This analysis showed for most antibodies a trend for positive antigenic selection in the CDRs of heavy and/or light chains, which was comparable to that observed in virus-neutralizing antibodies (Supplemental Table 1 and data not shown). Alignment of the H-CDR3 sequences did not reveal shared motifs, but identified an aromatic cluster (YYYYF) at the tip of H-CDR3 
A
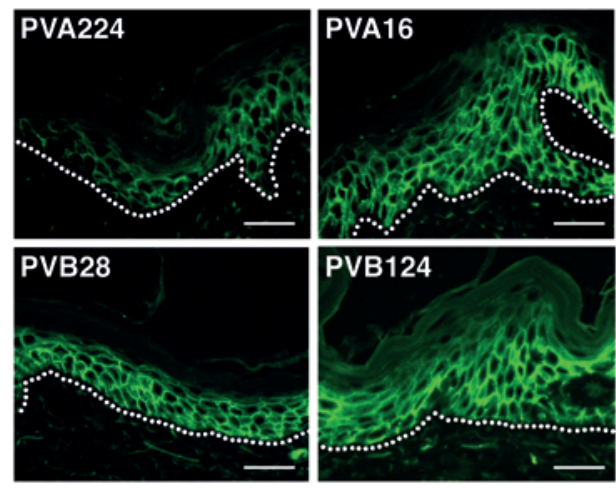

PVB124
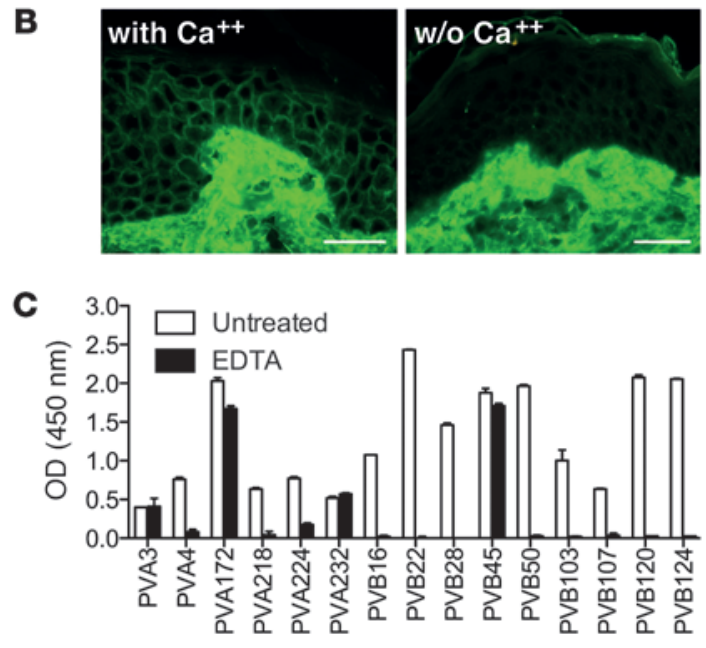

of the PVA224 antibody (Supplemental Figure 2). Taken together, our data indicate that in PV patients, the DSG3 antibody response is polyclonal, is not biased toward a particular $V$ gene, and is likely to be antigen selected.

DSG3 subdomain specificity and pathogenic activity of autoantibodies. To grossly map the targets of the autoantibodies isolated, we used chimeric DSG2 molecules containing DSG3 subdomains (20) and measured the ability of these molecules to inhibit binding of the autoantibodies to DSG3-coated plates. Using this assay, we found that the antibodies from patient PVA mapped to different DSG3 subdomains (EC1, EC2, EC3, or EC5), while 7 out of the 9 antibodies from patient PVB targeted the EC2 subdomain and 2 recognized the EC5 subdomain (Figure $2 \mathrm{~A}$ ). The high prevalence of EC2-specific antibodies isolated from the PVB patient was paralleled by the predominant reactivity of PVB serum with EC2 in the same assay (Figure 2B).

\section{Figure 2}

DSG3 subdomain specificity of DSG3-specific autoantibodies. (A) Inhibition of mAb binding to coated DSG3 by chimeric molecules consisting of DSG2 carrying a single DSG3 subdomain (EC1, EC2, EC3, EC4, or EC5). Black squares indicate a significant inhibition of antibody binding. Two mouse mAbs (mu-mAb), AK23 and RD, specific for EC1 and EC5, respectively, were also analyzed. Results are representative of 2 independent experiments. (B) Inhibition of PVA and PVB serum antibody binding to coated DSG3 by chimeric DSG2-DSG3 molecules carrying the indicated DSG3 subdomains. Shown are mean values \pm SEM of 2 independent experiments.

\section{Figure 1}

Human antibodies bind conformational epitopes in the DSG3 ectodomain. (A) Cryosections of human skin were stained with biotinylated human mAbs isolated from PV patients. Shown are 4 representative antibodies out of the 15 analyzed. The cutaneous basement membrane zone is marked by a dotted line. Scale bars: $100 \mu \mathrm{m}$. (B) Staining of human epidermis performed as in A with antibody PVB28 in the presence (with) or absence (w/o) of $\mathrm{Ca}^{+}$. Scale bars: $100 \mu \mathrm{m}$. (C) Binding of the indicated antibodies to DSG3-coated plates in the presence or absence of EDTA as assessed by ELISA. Data are representative of 2 separate experiments (mean \pm SEM).

To evaluate the pathogenicity of the isolated antibodies, we used an in vitro keratinocyte dissociation assay (21). Incubation of a confluent monolayer of human keratinocytes with pathogenic antibodies in the presence of exfoliative toxin A (ETA), which specifically cleaves DSG1, resulted in the fragmentation of the keratinocyte sheet. The EC1-specific antibody PVA224 and, surprisingly, 2 EC2-specific antibodies (PVB28 and PVB124) were able to dissociate the monolayer to an extent comparable to that of the reference mouse antibody AK23, while other EC2-, EC3-, and EC5specific antibodies were not effective (Figure 3 , A and B).

To assess in vivo pathogenicity, we selected 2 antibodies (PVA224 and PVB28) that were found to crossreact with mouse DSG3 and tested them in a mouse model of passively transferred PV (4). When injected subcutaneously into newborn mice together with ETA, PVA224 and PVB28 induced macroscopic blisters, intraepidermal suprabasal blistering and acantholysis, and intercellular deposition of human IgG in the epidermis (Figure 3, C-E). As a control, antibodies PVB16 and PVB124, which do not recognize murine DSG3, did not cause macroscopic or microscopic blisters or IgG deposition in the epidermis. Taken together, the above findings indicate that pathogenic antibodies bind to either EC1 or EC2 subdomains and that, in addition to EC1-specific antibodies, a fraction of EC2-specific antibodies also have pathogenic activity.

Human pathogenic antibodies target the EC1-EC2 cis-adhesive DSG3 interface. To gain insight into the epitope specificity and the mechanism of action of pathogenic antibodies, we performed a cross-

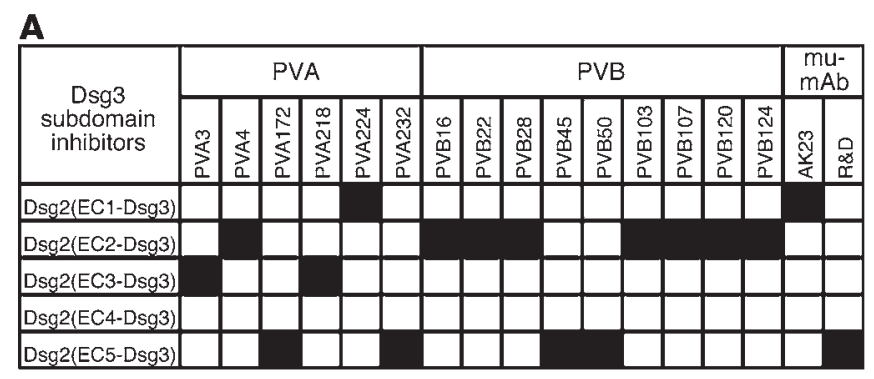

B

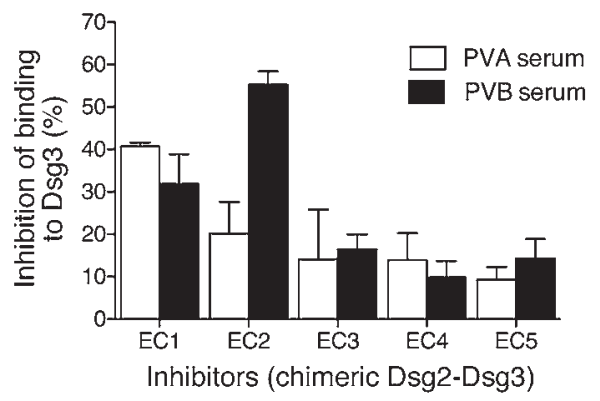


A
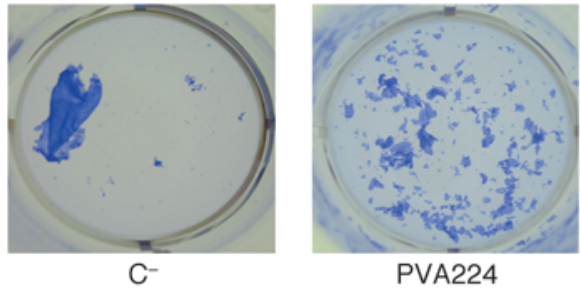

PVA224
B

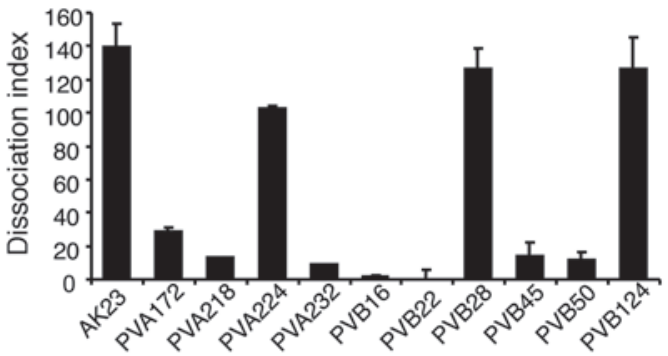

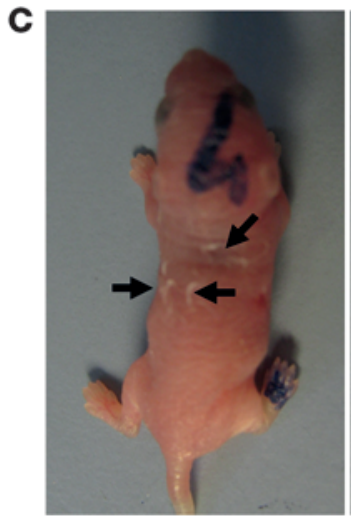
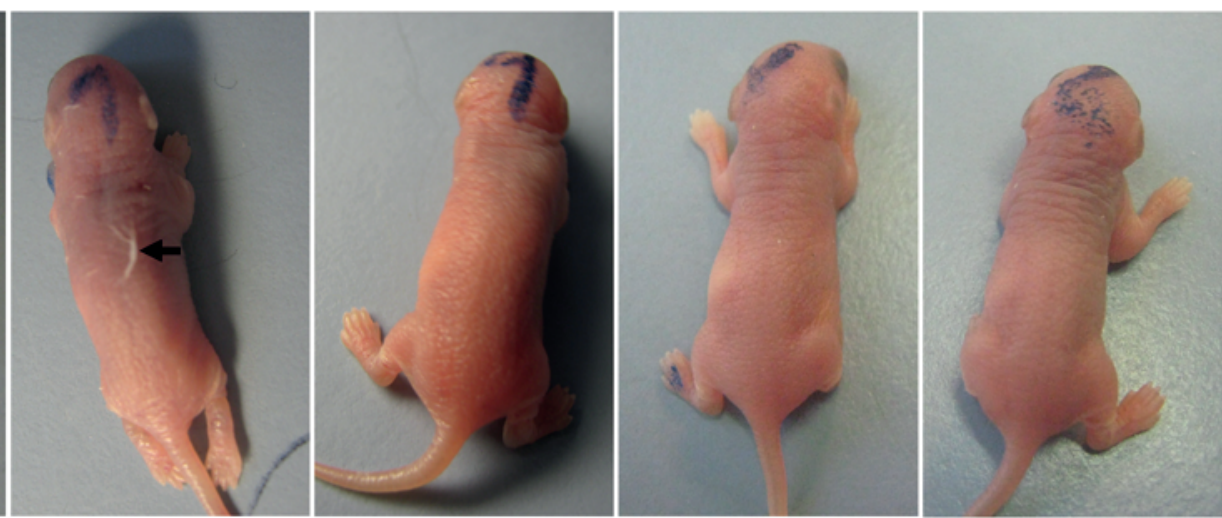

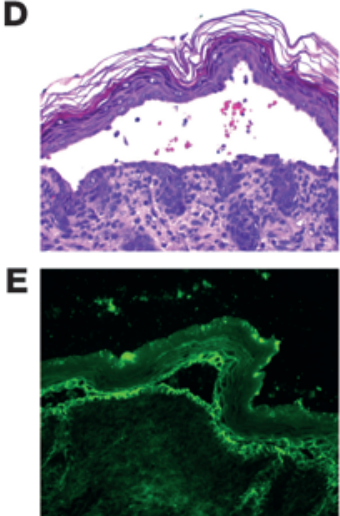

PVA224

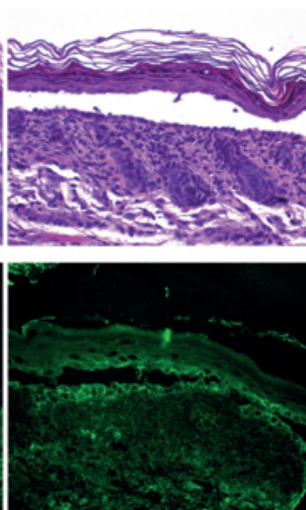

PVB28

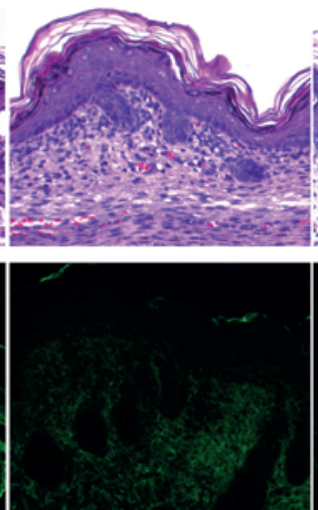

PVB124

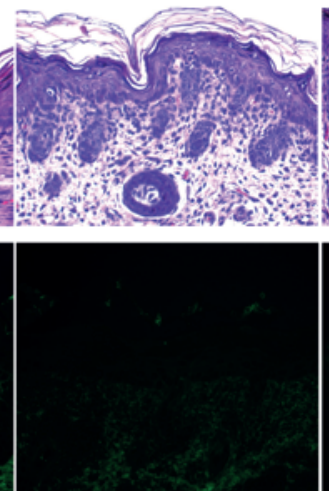

PVB16
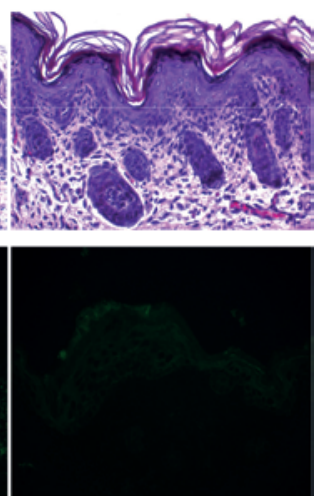

ETA

Figure 3

In vitro and in vivo pathogenic activity of anti-DSG3 antibodies. (A) Representative keratinocyte dissociation experiment. Primary human keratinocytes were seeded to confluence, and mAb PVA224 and a control antibody were added (1-10 $\mu \mathrm{g} / \mathrm{ml})$, followed by addition of ETA to cleave DSG1 molecules. Cells were incubated with dispase I to detach the monolayer from the plate, and sheet fragments were fixed and stained using crystal violet. (B) Keratinocyte dissociation induced by different antibodies. Shown is the dissociation index determined as specified in Methods in duplicate cultures in 2 independent experiments (mean \pm SEM). (C-E) Macroscopic blisters (black arrows) (C), intraepidermal suprabasal blistering and acantholysis (D), and intercellular deposition of human IgG in the epidermis (E) induced by injection into newborn mice of the indicated antibodies coadministered with ETA. Data are representative of results obtained from groups of 5 mice. Original magnification, $\times 200$.

competition assay for binding to DSG3-coated plates. Surprisingly, the pathogenic EC1-specific antibody PVA224 did not compete for binding with the mouse pathogenic antibody AK23, which has been shown to disrupt the EC1-EC1 trans-adhesion of DSG3 (refs. $8,9,11,13$, and Supplemental Figure 3, A and B). This finding indicates that the 2 pathogenic antibodies AK23 and PVA224 bind to nonoverlapping epitopes in the DSG3 EC1 domain. Consistently, PVA224 IP both the DSG3 mature form, in which the trans-adhesive interface is accessible, and the higher molecular weight DSG3 precursor, in which the trans-adhesive interface is not accessible, being masked by the presence of the propeptide (Supplemental
Figure 3E). In contrast, AK23 IP only the mature form of DSG3. Using the same competition assay, we found that the 2 EC2-specific pathogenic antibodies PVB28 and PVB124 cross-competed for binding to DSG3, indicating that they recognize the same or overlapping regions of DSG3 EC2 (Supplemental Figure 3C).

To identify the epitopes recognized by the 3 human pathogenic antibodies, we used PEPSCAN technology and screened libraries of DSG3 EC1 and EC2 peptides comprising 15-mer linear peptides, 17-mer peptides looped by a disulfide bond, and double CLIPS (Chemical Linkage of Peptides onto Scaffolds) peptides locked into double loops by 3 disulfide bonds. The EC1-specific antibody PVA224 was found 
A

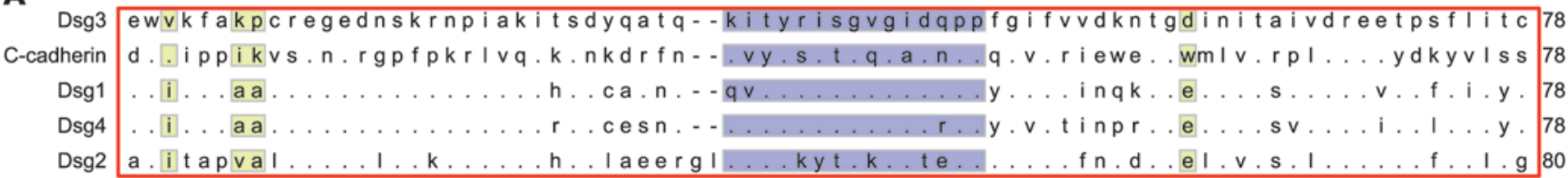

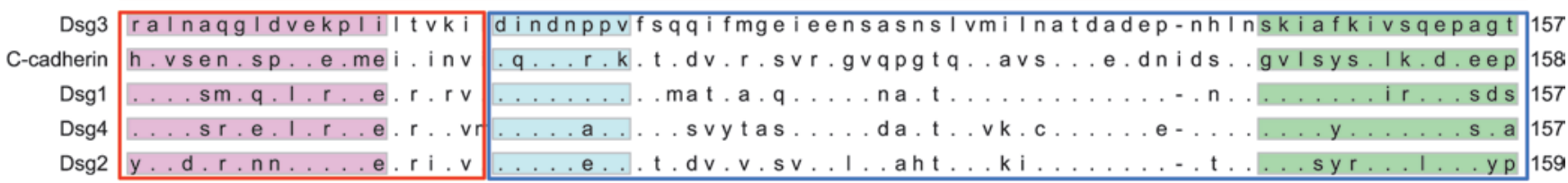

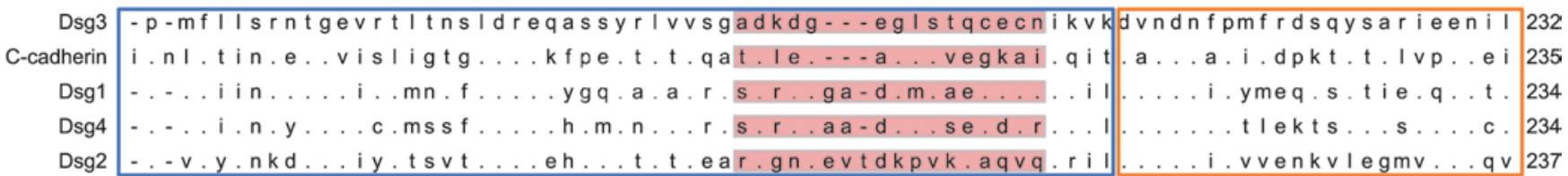

B

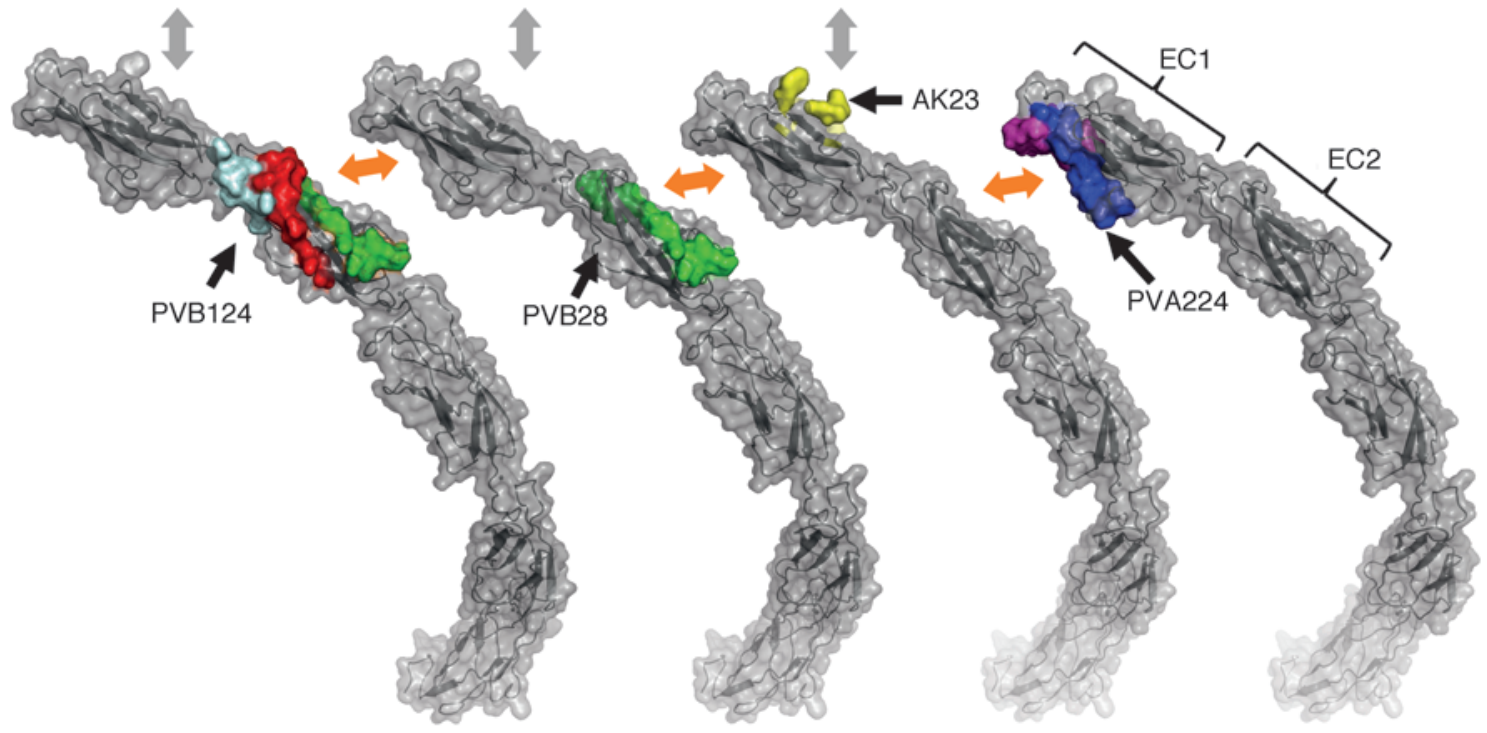

Figure 4

Epitope mapping of 3 pathogenic antibodies. (A) Alignment of DSG3 amino acid sequence 1-232 with C-cadherin, DSG1, DSG2, and DSG4. EC1, EC2, and EC3 subdomains are highlighted with red, blue, and orange boxes, respectively. Peptides recognized by PVA224 are highlighted in blue and pink, peptides recognized by PVB28 in green, and peptides recognized by PVB124 in light blue, red, and green. The reported amino acid residues bound by AK23 (13) are highlighted in yellow. (B) Location of peptides recognized by PVA224, PVB28, PVB124, and AK23 on the structure of C-cadherin ectodomain as determined in ref. 8 . Color code is as above. The trans-adhesive interface is indicated by a gray double arrow. The cis-adhesive interfaces are indicated by orange double arrows.

to recognize a peptide from the linear library (KITYRISGVGIDQPP) and a conformation-dependent peptide from the double CLIPS library (RALNAQGLDVEKPLI). Since structural information of the DSG3 domains is not available, we aligned the DSG3 sequence with that of C-cadherin, for which structural information is available (ref. 8 and Figure 4A). The positioning of the homologous peptides in the C-cadherin crystal structure defines the PVA224 epitope as a discontinuous epitope located in the EC1 region on the opposite side of the trans-adhesive interface recognized by AK23 (Figure 4B). According to this mapping, PVA224 is expected to disrupt the EC1-EC2 interface involved in cis-interaction of cadherin molecules required to form lateral strand dimers (8). Comparable results were obtained by modeling the peptides recognized by PVA224 on the DSG2 EC1 nuclear magnetic resonance (NMR) structure (data not shown).
The same approach was used to map the epitopes recognized by EC2-specific pathogenic antibodies PVB28 and PVB124. Peptides recognized by PVB28 and PVB124 were identified in the double CLIPS library. Both antibodies bound to a CLIPS peptide (SKIAFKIVSQEPAGT), while PVB124 bound also 2 amino acid stretches located at the N terminus (DINDNPPV) and at the C terminus (ADKDGEGLSTQCECN) of the EC2 subdomain (Figure $4 \mathrm{~B}$ ). The location of the homologous peptides on $\mathrm{C}$-cadherin defines 2 discontinuous overlapping epitopes in the EC2 region that are expected to interact in cis with the EC1 of the neighboring molecule (Figure 4B). The proximity of the 2 epitopes is consistent with finding that PVB28 and PVB124 cross-compete for binding to DSG3 (Supplemental Figure 3C). However, the ability of PVB28, and not of PVB124, to cross-react with mouse DSG3 indicates 
A
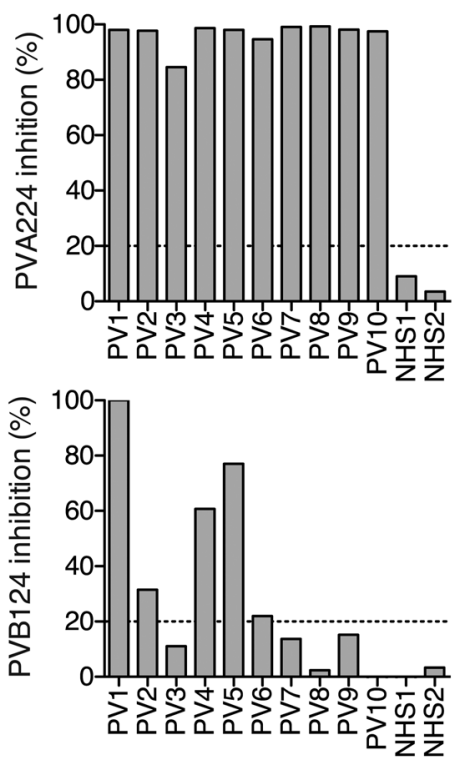

B

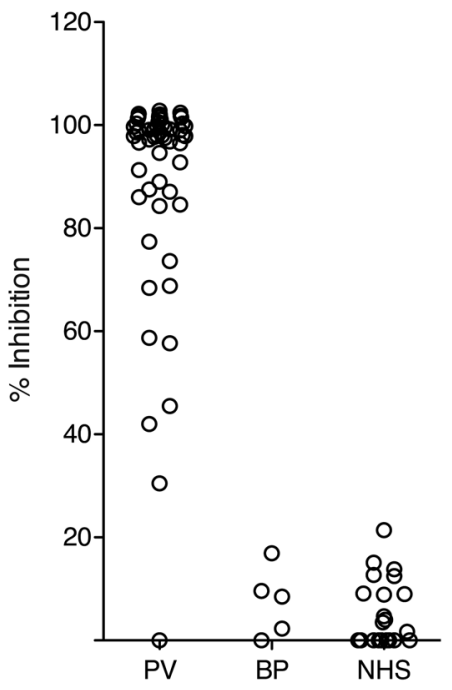

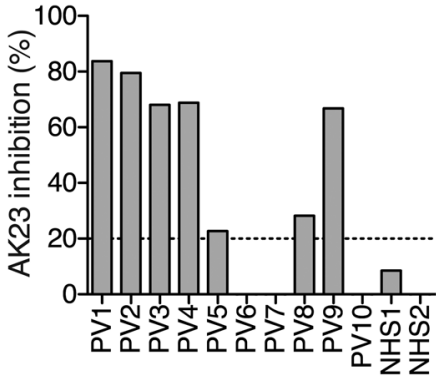

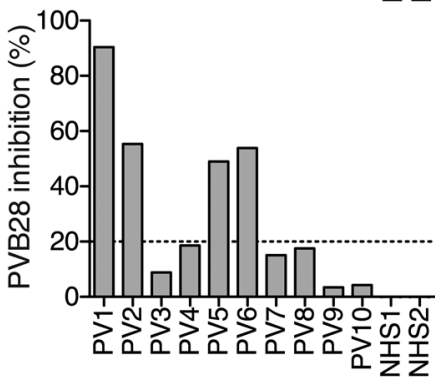

C

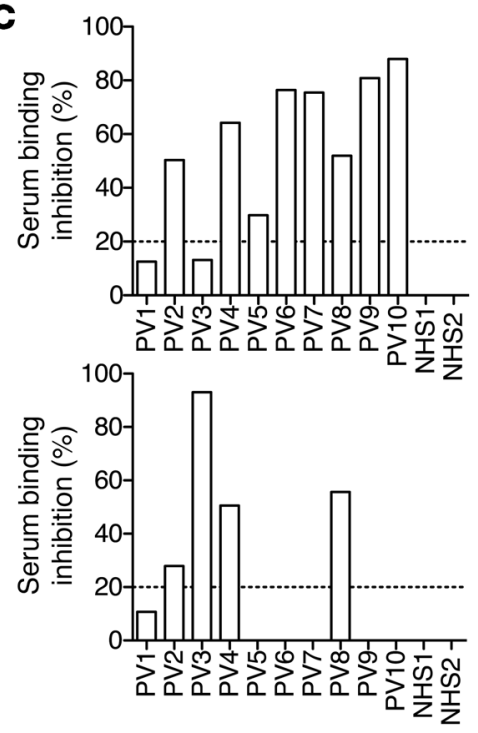

\section{Figure 5}

The PVA224 site on DSG3 EC1 is an immunodominant target for pathogenic autoantibodies in PV patients. (A) The inhibitory capacity of $10 \mathrm{PV}$ and 2 control sera on the binding of human (PVA224, PVB124, and PVB28) and mouse (AK23) pathogenic antibodies to DSG3. (B) The inhibitory capacity of patient or control sera to inhibit binding of PVA224 to DSG3. Sera from 57 PV patients, 10 bullous pemphigoid (BP) patients, and 32 healthy donors (NHS) are shown. (C) The inhibitory capacity of murinized PVA224 (top) or mouse AK23 (bottom) on the binding of serum antibodies from $10 \mathrm{PV}$ patients and 2 controls. Cut-off value (dashed line) is the mean of inhibition value of the 40 control sera plus 2 SD. Similar results were obtained in 2 independent experiments.

The high prevalence of PVA224-like antibodies in PV patients was further supported by analysis of sera from $57 \mathrm{PV}$ patients (Figure 5B). In contrast, such antibodies were absent in sera from patients with bullous pemphigoid and healthy donors.

To estimate the fraction of total serum DSG3specific antibodies targeting the PVA224 and AK23 sites, we measured the capacity of AK23 and of a "murinized" version of PVA224 to inhibit binding of human serum polyclonal antibodies to DSG3-coated plates. PVA224 inhibited binding of 8 out of 10 PV sera with values ranging from $20 \%$ to $90 \%$, while AK23 inhibited only 4 out of $10 \mathrm{PV}$ sera (Figure 5C). Taken together, the above results indicate that the PVA224 site on DSG3 EC1 is an immunodominant target for pathogenic autoantibodies in $\mathrm{PV}$ patients, while sites defined by AK23 on EC1 and by PVB124 and PVB28 on EC2 are subdominant, being targeted in some but not all patients.

Reversion of somatic mutations in VH but not VL genes abolishes binding to DSG3. To gain insights into the fundamental question on the nature of the immune response that led to the generation of pathogenic autoantibodies, we produced recombinant antibodies in which we removed all somatic mutations in the $\mathrm{VH}$ and VL genes of 3 pathogenic antibodies (PVA224,

that the 2 antibodies recognize overlapping but different epitopes. Taken together, the above data indicate that the 3 human pathogenic antibodies isolated target 2 novel sites on the EC1 and EC2 subdomains of DSG3 that are part of the cis-adhesive EC1-EC2 interface found in DSG3 arrays.

The PVA224 epitope is the main target of PV autoantibodies in human sera. To determine whether antibodies of the same specificity as the characterized antibodies were present in the sera of PV patients, we measured the capacity of serum antibodies to inhibit binding of human and mouse pathogenic antibodies to DSG3. ELISA plates were coated with DSG3 and incubated with PV or control sera, followed by the addition of biotinylated AK23, PVA224, PVB124, or PVB28 antibodies, which were then detected using enzyme-labeled streptavidin. All PV sera tested, but not control sera, inhibited binding of PVA224 by more than $80 \%$, consistent with the presence in the sera of antibodies against the cis-adhesive site targeted by PVA224 (Figure 5A). In contrast, only 5, 3, and 4 out of 10 sera inhibited binding of AK23, PVB124, and PVB28, respectively, by at least 50\%.
PVB28, and PVB124) and 1 nonpathogenic antibody (PVB16) and measured the capacity of these "germlined" antibodies (Supplemental Figure 4) to bind to DSG3. Strikingly and in contrast to the mutated antibodies, all the germlined antibodies failed to bind to DSG3 when tested by ELISA or IF on DSG3 transfectants or human skin (Figure 6, A and B, and Supplemental Figure 5). In addition, recombinant antibodies with mutated $\mathrm{VH}$ but germlined $\mathrm{VL}$ were capable of binding to DSG3, as the original antibody indicating that binding of the tested antibodies to DSG3 required somatic mutation in the heavy but not in the light chain (Figure 6B). We conclude that in some antibodies isolated from PV patients, autoreactivity was acquired as a function of somatic mutation in the $\mathrm{VH}$ genes.

\section{Discussion}

In this study, we isolated pathogenic IgG autoantibodies from 2 PV patients and demonstrated that these antibodies target the EC1 and EC2 subdomains of DSG3 in regions that are involved in cis-adhesive interactions. The finding that EC2 is targeted by 
A
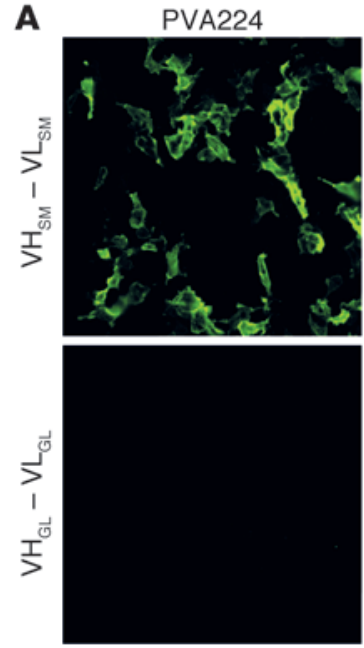

PVB28
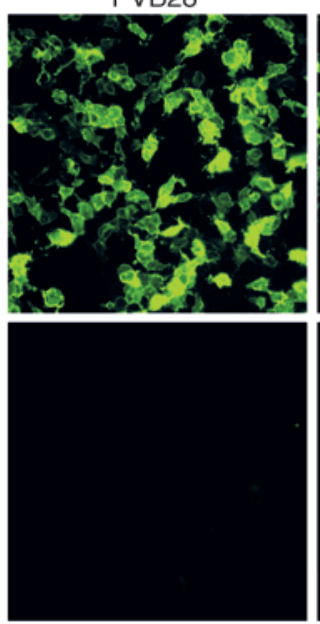

PVB124
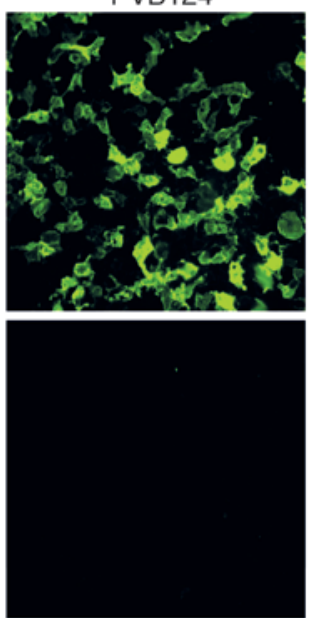

PVB16

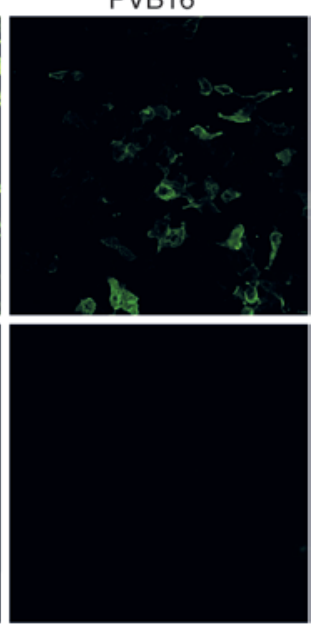

B
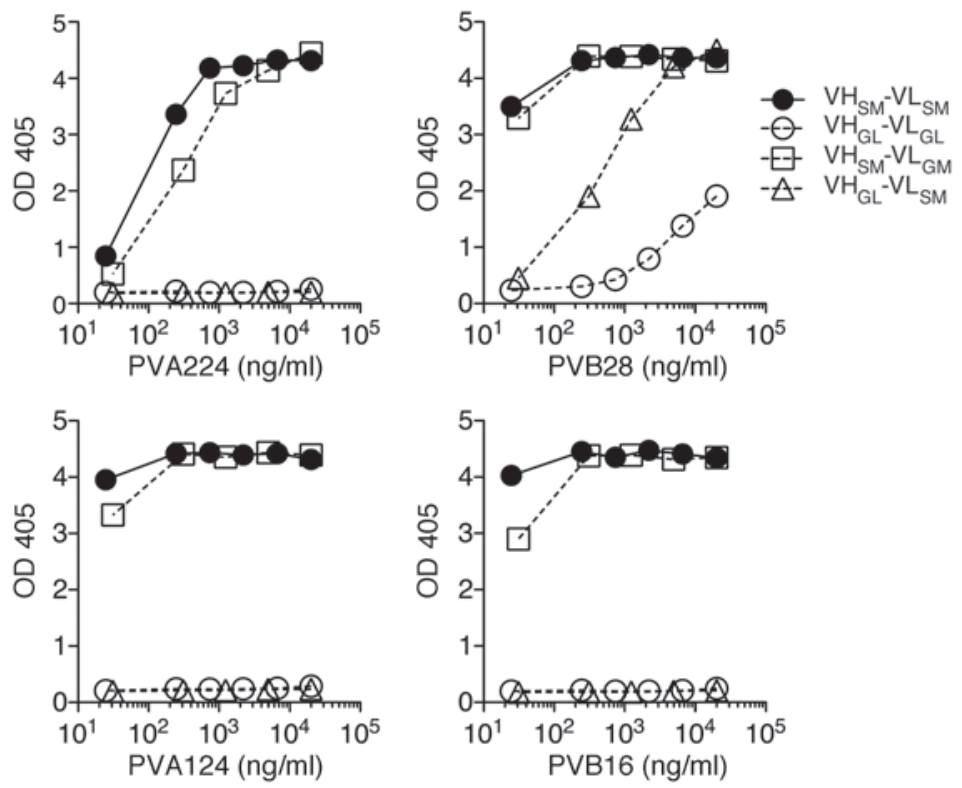

Figure 6

DSG3 autoreactivity is determined by somatic mutations in the $\mathrm{VH}$ genes. Four antibodies were produced recombinantly in the original VH/VL somatically mutated version $\left(\mathrm{VH}_{\mathrm{SM}}-\mathrm{VL}_{\mathrm{SM}}\right.$ ), in a full germlined $\mathrm{VH} / \mathrm{VL}$ version $\left(\mathrm{VH}_{\mathrm{GL}}-\mathrm{VL}_{\mathrm{GL}}\right)$, or a $\mathrm{VH}$ only or $\mathrm{VL}$ only germlined version $\left(\mathrm{VH} \mathrm{H}_{\mathrm{GL}}-\mathrm{V} \mathrm{L}_{S \mathrm{SM}}\right.$ or $\mathrm{VH}_{\mathrm{SM}}-\mathrm{VL}_{\mathrm{GL}}$, respectively). The antibodies were tested for staining of 293T cells transfected with DSG3 gene (A) and binding to DSG3-coated plates as assessed by ELISA (B). Representative experiment out 3 independent experiments performed. The low binding of the germlined PVB28 was also observed on uncoated plates and can be explained by a low degree of polyspecificity.

serum and pathogenic mAbs of pemphigus patients has been recently reported $(20,22)$, but the mAbs' fine specificity has not been defined. Previous studies in a mouse model of PV led to the isolation and characterization of a mouse pathogenic antibody, AK23, that binds to EC1 and disrupts the DSG3-DSG3 transadhesive interaction (13). Importantly, by analyzing PV sera for their capacity to inhibit binding to DSG3 of human and mouse pathogenic antibodies, we demonstrate that virtually all PV patients tested have serum antibodies to the cis-adhesive interface of EC1 (defined by PVA224), while a subset of patients have antibodies to the trans-adhesive interface of EC1 (defined by AK23) or to the cis-adhesive interface of EC2 (defined by PVB124). These findings not only identify an immunodominant site targeted by pathogenic PV autoantibodies, but also suggest a mechanism of blister formation in PV. Availability of DSG3 crystal structure and further functional studies are needed to confirm this hypothesis.

A body of experimental evidence supports the essential role of lateral cadherin dimerization, in addition to trans-interaction, in mediating cell-cell adhesion $(23,24)$. In addition, recent studies have shown that PV-IgG treatment of keratinocytes causes internalization of nondesmosomal DSG3 clusters, resulting in the formation of DSG3-depleted desmosomal complexes and loss of cell-cell adhesion (25-28). These observations are in line with initial electron microscopy studies demonstrating that keratinocytes separate first in the interdesmosomal areas of early PV lesions, when desmosomes are intact and still adhere to each other (29, 
30). Thus, it is tempting to suggest that pathogenic antibodies could interfere with DSG3-DSG3 cis-interaction in the nondesmosomal as well as in the desmosomal compartment.

Another major finding of this study is the demonstration, for what we believe is the first time in an organ-specific autoimmune disease, that the germline version of the autoantibodies does not recognize the nominal autoantigen. Furthermore, at variance with previous studies $(14,31)$, we show that the response is polyclonal and that the autoantibodies use different $\mathrm{VH}$ and VL and all IgG isotypes. The finding that replacing mutations are mainly found in the CDRs is suggestive of antigenic selection, and in the 4 pathogenic antibodies studied in more detail, we have shown that the replacing mutations responsible for DSG3 reactivity lay in the $\mathrm{VH}$, a finding that is reminiscent of a previous report describing a consensus sequence in the CDR3 of pathogenic antibodies (32).

The finding that germlined autoantibodies do not bind DSG3 suggests that this autoantigen does not represent the primary trigger of the pathogenic B cell clones. Somatic mutations are known to expand the $\mathrm{B}$ cell repertoire by intraclonal B cell receptor (BCR) diversification in the memory compartment (33). A role for somatic mutation in development of autoantibodies has been initially demonstrated in the mouse system $(34,35)$, and more recent studies using germlined antibodies have extended these observations to the human system. In particular, 2 anti-DNA autoantibodies isolated from SLE patients were found to be derived from nonautoreactive B cells, suggesting that they developed as a byproduct of a normal immune response (36). Furthermore, it was shown that in healthy individuals, a substantial fraction of IgG memory B cells express self-reactive and polyreactive specificities that develop by somatic mutations and are absent in bone marrow plasma cells $(37,38)$. We found that, with the exception of PVB28, the germlined antibodies were not polyreactive, suggesting that their selection was not driven by heteroligation, as suggested for HIV-neutralizing antibodies (39). However, we cannot rule out the possibility that the germlined antibodies might have an affinity for DSG3 sufficient to trigger naive B cells, but insufficient to be detected in classical binding assays.

The derivation of autoantibodies from an ongoing immune response to an unrelated antigen through fortuitous mutations introducing self reactivity represents a plausible mechanism for the generation of humoral autoimmunity. The low activation threshold of activated and memory B cells and their capacity to proliferate and differentiate in the absence of $\mathrm{T}$ cell help $(40,41)$ may be the basis for the selection of autoreactive clones. The finding that PV autoantibodies characterized in this study belong to all IgG classes, in particular IgG4, would be consistent with their derivation from distinct types of ongoing chronic immune responses that are known to elicit IgG4 antibody production (42). However, it is also possible that the pathogenic antibodies might have derived from the antibody response to a particular foreign antigen.

In conclusion, the characterization of pathogenic mAbs points to the disruption of DSG3 cis-interaction as a possible mechanism underlying blister formation in PV. The identification of an immunodominant region targeted by pathogenic antibodies has implications for diagnosis of $\mathrm{PV}$ and opens new perspectives toward the establishment of therapeutic approaches for treatment of PV patients. Finally, the germlined version of the PV autoantibodies may lead to the identification of the antigens that eventually lead to development of this life-threatening disease.

\section{Methods}

Isolation of monoclonal autoantibodies from PV patients. Peripheral blood samples were obtained from 2 patients (PVA and PVB) suffering from active mucocutaneous PV. The patients showed typical clinical, histological, and immunopathological features and had high-titer anti-DSG circulating autoantibodies (PVA: DSG3, 308 U/ml, DSG1, 110 U/ml; PVB: DSG3, 191 $\mathrm{U} / \mathrm{ml}$, DSG1, $170 \mathrm{U} / \mathrm{ml}$ ), as assessed by ELISA kits based on ectodomain of DSG1 and DSG3 (MBL). IgG ${ }^{+}$memory B cells were isolated from cryopreserved PBMC using CD22 microbeads (Miltenyi Biotec) followed by depletion of cells carrying IgM, IgD, and IgA by cell sorting. Multiple replicate microcultures of $10-30 \mathrm{IgG}^{+}$memory B cells/well (for a total of 2 to $8 \times 10^{4}$ purified cells) were infected with EBV and CPG as previously described (15). Culture supernatants were tested for binding to DSG3-coated ELISA plates and for binding to the keratinocyte cell line (HaCaT) monolayers by IF assay using an automated fluorescence microscope (Pathway 855; BD). The specificity of positive polyclonal cultures was further assessed by IF on primary human keratinocytes and by ELISA on recombinant DSG1. Positive reactivities detected $(0.17 \%$ and $2.12 \%$ of memory B cell seeded for PVA and PVB, respectively) were confirmed by the propagation of oligoclonal cultures. Positive cultures were cloned by limiting dilution and expanded; antibodies were purified using protein $G$ columns. Specific $m A b s$ could be isolated only from a fraction of the initial hits (12\% in PVA and $20 \%$ in PVB).

IF studies. Supernatants from immortalization cultures or cloned B cells were tested by IF staining for their binding to live $\mathrm{HaCaT}$ and primary human keratinocytes. Cells were seeded at $2 \times 10^{4} \mathrm{cells} / \mathrm{cm}^{2}$ in flat-bottom 384- or 96-well plates and were grown to confluence. Confluent monolayers were grown for an additional 16 hours in fresh medium supplemented with $1.2 \mathrm{mM} \mathrm{CaCl}_{2}$. IF staining was performed on cell monolayers in their culture plates. Cells were washed in PBS and incubated for 0.5 hours at room temperature (RT) with B cell culture supernatants, washed, and stained for an additional 0.5 hours with Alexa Fluor 488-conjugated goat anti-human IgG (Invitrogen). Human antibodies of irrelevant specificity were used as negative controls. Murine AK23 antibody, revealed by Alexa Fluor 488-conjugated goat anti-mouse IgG, was used as a positive control. Serial images of stained keratinocyte monolayers were acquired by the BD Pathway 855 automated fluorescence microscope. Staining was also performed on cryosections of monkey esophagus and human or mouse skin. Cryosections of skin of neonatal mice injected with pathogenic antibodies were blocked with PBS containing 3\% BSA and stained with FITC-conjugated rabbit anti-human IgG (DAKO).

ELISA assays. Recombinant proteins DSG3(aa 1-566) and DSG1(aa 1-496) were produced in baculovirus and affinity purified as previously reported (2). ELISA plates were coated with DSG1 and DSG3, blocked with $1 \% \mathrm{BSA}$, and incubated with antibodies followed by HRP-conjugated antihuman IgG Fcy fragment-specific secondary antibodies (Jackson ImmunoResearch). For mapping of calcium-dependent conformational epitopes, domain-swapped DSG3 recombinant molecules (DSG3 EC1-EC5 in the backbone of DSG2) were used $(20,43)$. Limiting antibody concentrations were incubated with an excess of baculovirus culture supernatant containing a single swapped molecule and tested for their capacity to bind to DSG3-coated plates. To measure sensitivity to calcium depletion, wells coated with DSG3 were incubated in the presence or absence of $10 \mathrm{mM}$ EDTA and developed as described above. To determine the epitope specificity of serum antibodies, sera from PV patients or controls were tested at a fixed dilution (1:25) for their capacity to inhibit binding of limiting amounts of biotinylated human antibodies (or mouse AK23 or murinized PVB224 antibodies) to DSG3-coated plates. The capacity of mAbs to inhibit binding of polyclonal serum antibodies was measured by adding to DSG3 ELISA plates murinized PVA224 or AK23 antibodies first, followed by PV sera and anti-human secondary reagents. The cut-off value was calculated as the 
mean of inhibition value of the 30 control (normal human serum [NHS]) and 10 sera from bullous pemphigoid patients plus 2 SDs. The competition between unlabeled and biotinylated or "murinized" mAbs was measured by adding to DSG3-coated plates first unlabeled competitor antibodies at different concentrations, followed by the labeled antibodies added at a concentration corresponding to $70 \%-80 \%$ of the maximal OD level. Bound labeled antibodies were detected using alkaline phosphatase-labeled (AP-labeled) streptavidin (for biotynilated antibody) or AP-labeled anti-mouse IgG (Jackson Immunoresearch). Samples were tested in duplicates, and percentage of inhibition was calculated as follow: (1 - [(OD sample - OD negative control $) /$ $(O D$ positive control $-O D$ negative control $)]) \times 100$.

Immunoprecipitation studies. In selected cases, mapping results by ELISA were confirmed by IP studies. Briefly, baculovirus culture supernatants containing specific domain-swapped baculoproteins (DSG3 EC1-EC5/ DSG2) and DSG2 and DSG3 as controls were incubated at RT for 1 hour with each antibody. As all the constructs were E-tagged, and an anti-E-tag polyclonal antibody (Bethyl) was used as a positive control. The proteinantibody complex was incubated with protein A/G PLUS Agarose (Santa Cruz Biotechnology Inc.) at $4^{\circ} \mathrm{C}$ overnight. Centrifuged IPs were applied to SDS-PAGE and blotted onto a polyvinylidene difluoride membrane (Millipore). To detect IP recombinant proteins, membranes were first incubated with the anti-E-tag rabbit polyclonal antibody and then with a secondary AP-conjugated anti-rabbit IgG antibody (SouthernBiotech). The filter was washed and stained with $330 \mathrm{mg} / \mathrm{ml}$ of nitroblue tetrazolium (NBT) and $165 \mathrm{mg} / \mathrm{ml}$ of 5-bromo-4-chloro-3-indolyl phosphate (BCIP) (Roche Diagnostics Corp.) in substrate buffer $(100 \mathrm{mM} \mathrm{NaCl}, 100 \mathrm{mM}$ Tris- $\mathrm{HCl}, \mathrm{pH}$ 9.6, $5 \mathrm{mM} \mathrm{MgSO}_{4}$ ). The same IP procedure was applied to IP the precursor and/or mature form of DSG3 using recombinant baculoprotein produced and affinity purified as previously reported (2).

Antibody purification, labeling, genetic analysis, and reversion to germline. Human $\mathrm{mAbs}$ were affinity purified by protein A or protein $\mathrm{G}$ chromatography (GE Healthcare) and desalted against PBS. Selected antibodies were biotinylated using the EZ-Link NHS-PEO Solid Phase Biotinylation Kit (Pierce). The "murinization" of human antibodies was performed by cloning the $\mathrm{VH}$ and $\mathrm{VL} / \mathrm{VK}$ genes into expression vectors containing human IgG1, Igא, and Ig $\lambda$ constant regions in which the human constant region (hinge, $\mathrm{CH} 2$, and $\mathrm{CH} 3$ domains) was replaced by the homologous mouse IgG2a. Recombinant antibodies were produced by transient transfection of 293 Freestyle Cells (Invitrogen) using polyethylenimine (PEI). The usage of $\mathrm{VH}$ and $\mathrm{VL}$ gene segments was determined by sequencing, and analysis for homology to known human $\mathrm{V}, \mathrm{D}$, and J genes was performed using the IMGT (international ImMunoGeneTics information system) database (44). The analysis for the presence of positive or negative antigenic selection for $\mathrm{VH}$ and $\mathrm{VK} / \mathrm{VL}$ mutated gene segments was performed using both the Focused and Global tests for comparison (http://clip.med.yale. edu/selection), as described (18). Germlined sequences were determined by reverting mutations to the germline sequence while retaining the original CDR3 junctions and terminal deoxy-nucleotidyl transferase (TdT) N nucleotides. Germlined VH and VL nucleotide sequences were synthesized by Genscript, and their accuracies were confirmed by sequencing.

Epitope mapping. Overlapping linear 15-mer, cyclized 17-mer, and double CLIPS locked into double loops by 3 disulfide bond peptides based on EC1 and EC2 DSG3 were synthesized on polypropylene support (minicards) and were tested for reactivity with antibodies as described (45). Pepscan peptide-binding analysis was carried out by an ELISA-based for- mat in which the colored substrate was quantified with a charge-coupled device (CCD) camera and an image-processing system. The values mostly ranged from 0 to 3,000, a log scale similar to 1 to 3 of a standard 96-well plate ELISA reader.

Keratinocyte dissociation assay. The ability of antibodies to dissociate keratinocyte monolayers was measured according to Ishii et al (21). Primary human keratinocytes were seeded onto 12-well plates in KGM medium (Lonza) containing $0.15 \mathrm{mM} \mathrm{CaCl}_{2}$. Forty-eight hours after confluence, the medium was changed to $1.2 \mathrm{mM} \mathrm{CaCl}_{2}$ and $\mathrm{mAbs}$ were added $(1-10 \mu \mathrm{g} / \mathrm{ml})$ at $37^{\circ} \mathrm{C}$ overnight followed by addition of ETA $(1 \mu \mathrm{g} / \mathrm{ml})$ to cleave DSG1 molecules. After washing, the cells were incubated with dispase I (Roche Diagnostics Corp) for 30 minutes to detach the monolayer from the plate. Released cell sheets were washed with PBS twice and subject to mechanical stress by pipetting. The sheet fragments were fixed by adding formaldehyde at a final concentration of $3 \%$ and stained using crystal violet. The number of fragments was determined as an average of the number of fragments counted by using 3 sets of images captured by a digital camera for each plate. The dissociation index was calculated with the following formula: $\left(\mathrm{Ab}-\mathrm{C}^{-}\right)$/ $\left(\mathrm{C}^{+}-\mathrm{C}^{-}\right) \times 100$, where $\mathrm{C}^{+}$is $\mathrm{AK} 23$ and $\mathrm{C}^{-}$an irrelevant control antibody.

Passive transfer model. The pathogenic activity of antibodies was evaluated by passive transfer in neonatal mice (4). Human antibodies ( $300 \mu \mathrm{g} /$ mouse) were injected subcutaneously into the backs of neonatal C57BL/6NCrl mice together with $2 \mu \mathrm{g}$ ETA, which specifically cleaves DSG1. Controls were injected with the same dose of normal human IgG, PBS, or ETA alone. Mice were sacrificed 18 to 24 hours after injection. Skin was harvested for direct IF analysis and for histology.

Statistics. $P$ value cutoff used to determine statistical significance in the analysis for positive or negative antigenic selection using the Focus and Global binomial tests was 0.05 . Data were analyzed using GraphPad Prism software.

Study approval. The study was conducted in accordance with the Declaration of Helsinki guidelines and was approved by the IDI-IRCCS Ethics Committee; all patients gave informed consent.

\section{Acknowledgments}

We thank Naomi De Luca and Caterina Cattani for their technical assistance in immunohistochemistry and immunofluorescence microscopy. This work was supported by grants from the European Community's FP7 (Coordination Theme 1-HEALTH-F2-2008-200515 to M. Hertl, A. Lanzavecchia, and G. Zambruno), the Italian Ministry of Health (RF-20102309790 to G. Zambruno), and the Swiss National Science Foundation (31003A-126027 to A. Lanzavecchia). A. Lanzavecchia is supported by the Helmut Horten Foundation.

Received for publication April 19, 2012, and accepted in revised form July 12, 2012.

Address correspondence to: Giovanna Zambruno, Molecular and Cell Biology Laboratory, Istituto Dermopatico dell'Immacolata, Via Monti di Creta 104, 00167, Roma, Italy. Phone: 39.06.66464738; Fax: 39.06.66464738; E-mail: g.zambruno@idi.it. Or to: Antonio Lanzavecchia, Institute for Research in Biomedicine, Via V. Vela 6, 6500, Bellinzona, Switzerland. Phone: 41.91.8200310; Fax: 41.91.8200312; E-mail: lanzavecchia@irb.usi.ch.
1. Amagai M, Klaus-Kovtun V, Stanley JR. Autoantibodies against a novel epithelial cadherin in pemphigus vulgaris, a disease of cell adhesion. Cell. 1991;67(5):869-877.

2. Ishii $\mathrm{K}$, et al. Characterization of autoantibod- ies in pemphigus using antigen-specific enzymelinked immunosorbent assays with baculovirusexpressed recombinant desmogleins. J Immunol. 1997;159(4):2010-2017.

3. Ruach M, Ohel G, Rahav D, Samueloff A. Pemphi- gus vulgaris and pregnancy. Obstet Gynecol Surv. 1995;50(10):755-760

4. Anhalt GJ, et al. Induction of pemphigus in neonatal mice by passive transfer of IgG from patients with the disease. NEngl J Med. 1982;306(20):1189-1196. 
5. Amagai M, Koch PJ, Nishikawa T, Stanley JR. Pemphigus vulgaris antigen (desmoglein 3 ) is localized in the lower epidermis, the site of blister formation in patients. J Invest Dermatol. 1996;106(2):351-355.

6. Shimizu H, et al. Pemphigus vulgaris and pemphigus foliaceus sera show an inversely graded binding pattern to extracellular regions of desmosomes in different layers of human epidermis. J Invest Dermatol. 1995;105(2):153-159.

7. Ding X, et al. Mucosal and mucocutaneous (generalized) pemphigus vulgaris show distinct autoantibody profiles. J Invest Dermatol. 1997;109(4):592-596.

8. Boggon TJ, et al. C-cadherin ectodomain structure and implications for cell adhesion mechanisms. Sci ence. 2002:296(5571):1308-1313.

9. Sharma PM, et al. Pathogenic anti-desmoglein MAbs show variable ELISA activity because of preferential binding of mature versus proprotein isoforms of desmoglein 3. J Invest Dermatol. 2009;129(9):2309-2312.

10. Futei $Y$, et al. Use of domain-swapped molecules for conformational epitope mapping of desmoglein 3 in pemphigus vulgaris. J Invest Dermatol. 2000;115(5):829-834

11. Sekiguchi $M$, et al. Dominant autoimmune epitopes recognized by pemphigus antibodies map to the N-terminal adhesive region of desmogleins. J Immunol. 2001;167(9):5439-5448.

12. Amagai $M$, et al. Use of autoantigen-knockout mice in developing an active autoimmune disease model for pemphigus. J Clin Invest. 2000;105(5):625-631.

13. Tsunoda $\mathrm{K}$, et al. Induction of pemphigus phenotype by a mouse monoclonal antibody against the amino-terminal adhesive interface of desmoglein 3 . J Immunol. 2003;170(4):2170-2178.

14. Payne AS, et al. Genetic and functional characterization of human pemphigus vulgaris monoclonal autoantibodies isolated by phage display. J Clin Invest. 2005;115(4):888-899.

15. Traggiai E, et al. An efficient method to make human monoclonal antibodies from memory B cells: potent neutralization of SARS coronavirus. Nat Med. 2004;10(8):871-875.

16. Bhol K, et al. Correlation of peptide specificity and IgG subclass with pathogenic and nonpathogenic autoantibodies in pemphigus vulgaris: a model for autoimmunity. Proc Natl Acad Sci U S A. 1995;92(11):5239-5243.

17. Müller R, et al. IgG against extracellular subdomains of desmoglein 3 relates to clinical phenotype of pemphigus vulgaris. Exp Dermatol. 2008;17(1):35-43.

18. Uduman M, et al. Detecting selection in immuno- globulin sequences. Nucleic Acids Res. 2011;39(Web Server issue):W499-W504.

19. Hershberg U, Uduman M, Shlomchik MJ, Kleinstein $\mathrm{SH}$. Improved methods for detecting selection by mutation analysis of $\mathrm{Ig} \mathrm{V}$ region sequences. Int Immunol. 2008;20(5):683-694.

20. Ohyama B, et al. Epitope spreading is rarely found in pemphigus vulgaris by large-scale longitudinal study using desmoglein 2-based swapped molecules. I Invest Dermatol. 2012;132(4):1158-1168.

21. Ishii $\mathrm{K}$, et al. In vitro keratinocyte dissociation assay for evaluation of the pathogenicity of antidesmoglein $3 \mathrm{IgG}$ autoantibodies in pemphigus vulgaris. J Invest Dermatol. 2005;124(5):939-946.

22. Saleh MA, et al. Pathogenic anti-desmoglein 3 mAbs cloned from a paraneoplastic pemphigus patient by phage display. J Invest Dermatol. 2012;132(4):1141-1148.

23. Brieher WM, Yap AS, Gumbiner BM. Lateral dimerization is required for the homophilic binding activity of C-cadherin. J Cell Biol. 1996;135(2):487-496.

24. Yap AS, Brieher WM, Pruschy M, Gumbiner BM. Lateral clustering of the adhesive ectodomain: a fundamental determinant of cadherin function. Curr Biol. 1997;7(5):308-315.

25. Aoyama Y, Kitajima Y. Pemphigus vulgaris-IgG causes a rapid depletion of desmoglein 3 (Dsg3) from the Triton X-100 soluble pools, leading to the formation of Dsg3-depleted desmosomes in a human squamous carcinoma cell line, DJM-1 cells. J Invest Dermatol. 1999;112(1):67-71.

26. Sato M, Aoyama Y, Kitajima Y. Assembly pathway of desmoglein 3 to desmosomes and its perturbation by pemphigus vulgaris-IgG in cultured keratinocytes, as revealed by time-lapsed labeling immunoelectron microscopy. Lab Invest. 2000;80(10):1583-1592

27. Delva E, et al. Pemphigus vulgaris IgG-induced desmoglein-3 endocytosis and desmosomal disassembly are mediated by a clathrin- and dynamin-independent mechanism. J Biol Chem. 2008;283(26):18303-18313.

28. Jennings JM, et al. Desmosome disassembly in response to pemphigus vulgaris IgG occurs in distinct phases and can be reversed by expression of exogenous Dsg3. J Invest Dermatol. 2011;131(3):706-718.

29. Wilgram GF, Caulfield JB, Lever WF. An electron microscopic study of acantholysis in pemphigus vulgaris. J Invest Dermatol. 1961;36:373-382.

30. Hashimoto K, Lever WF. The intercellular cement in pemphigus vulgaris, an electron microscopic study. Dermatologica. 1967;135(1):27-34

31. Yamagami J, Takahashi H, Ota T, Amagai M.
Genetic characterization of human Dsg3-specific $B$ cells isolated by flow cytometry from the peripheral blood of patients with pemphigus vulgaris. J Dermatol Sci. 2008;52(2):98-107.

32. Yamagami J, et al. Homologous regions of autoantibody heavy chain complementarity-determining region 3 (H-CDR3) in patients with pemphigus cause pathogenicity. J Clin Invest. 2010;120(11):4111-4117.

33. Rajewsky K. Clonal selection and learning in the antibody system. Nature. 1996;381(6585):751-758.

34. Shlomchik MJ, Aucoin AH, Pisetsky DS, Weigert MG. Structure and function of anti-DNA autoantibodies derived from a single autoimmune mouse. Proc Natl Acad Sci U S A. 1987;84(24):9150-9154.

35. Pewzner-Jung Y, Simon T, Eilat D. Structural elements controlling anti-DNA antibody affinity and their relationship to anti-phosphorylcholine activity. J Immunol. 1996;156(8):3065-3073.

36. Wellmann U, et al. The evolution of human antidouble-stranded DNA autoantibodies. Proc Natl Acad Sci U S A. 2005;102(26):9258-9263.

37. Tiller T, et al. Autoreactivity in human IgG+ memory B cells. Immunity. 2007;26(2):205-213.

38. Scheid JF, et al. Differential regulation of self-reactivity discriminates between IgG+ human circulating memory B cells and bone marrow plasma cells. Proc Natl Acad Sci U S A. 2011;108(44):18044-18048.

39. Mouquet $\mathrm{H}$, et al. Polyreactivity increases the apparent affinity of anti-HIV antibodies by heteroligation. Nature. 2010;467(7315):591-595.

40. Bernasconi NL, Traggiai E, Lanzavecchia A. Maintenance of serological memory by polyclonal activation of human memory B cells. Science. 2002;298(5601):2199-2202

41. Hebeis BJ, et al. Activation of virus-specific memory B cells in the absence of T cell help. J Exp Med. 2004;199(4):593-602.

42. Aalberse RC, van der Gaag R, van Leeuwen J. Serologic aspects of IgG4 antibodies. I. Prolonged immunization results in an IgG4-restricted response. J Immunol. 1983;130(2):722-726.

43. Chan PT, et al. Immune response towards the amino-terminus of desmoglein 1 prevails across different activity stages in nonendemic pemphigus foliaceus. Br J Immunol. 2010;162(6):1242-1250

44. Lefranc MP, et al. IMGT, the international ImMunoGeneTics information system. Nucleic Acids Res. 2009;37(Database issue):D1006-D1012.

45. Timmerman P, Puijk WC, Meloen RH. Functional reconstruction and synthetic mimicry of a conformational epitope using CLIPS technology. J Mol Recognit. 2007;20(5):283-299. 